und Morgens, sondern auch zuweilen am heissen Tage pflügen sie mit ihrem Schnabel das Wasser. Im Ende Mai bekam ich noch nicht flugbare Junge von einer Sandbank bei Loko. Die Mägen der erlegten enthielten immer Fische.

185. Sterna minuta L.

Im unteren Niger, bei Lokodscha bis Loko am Benuë.

186. Struthio camelus L.

Soll nördlich von Sokoto wild leben. Ich habe ihn nur in gefangenem Zustande gesehen. Die Federn bilden einen Ausfubrartikel.

187. Bucorax abyssinicus $\mathrm{Gm}$.

Diese Art ist in der Aufählung vergessen worden und füge ich sie hier an, da ich sie im Benuë und bei Panda sah. In Loko wurde ein Exemplar, das in der Nähe gefangen war, lebend gebalten. -

\title{
Zur Erinnerung
}

an

\section{Gustav Adolf Fischer.}

Kaum ist das Trauergeläut verklungen, kaum verhallt die laute Klage um einen unserer begabtesten und eifrigsten Afrikaforscher, da greift das unerbittliche Schicksal von neuem mit rauher Hand in unsere Reihen - und einen Mann hat es hinweggerissen, an dessen Wirken wir nicht nur die Hoffnungen einer vielversprechenden Zukunft setzten, welcher vielmehr erprobt war in langjähriger Thätigkeit, welcher sich bewährt hatte in schwierigen Aufgaben, die er als Lebenszweck mit kühner Energie selbst sich vorgezeichnet. Einen Mann hat es getroffen, der auf der Höhe des Schaffens stand, welcher hinweisen konnte auf eine Reihe ruhmvoller Thaten, die er im Dienste der Wissenschaft vollführt, der in zehnjährigem Ringen einen ehrenvollen Platz unter den Naturforschern sich gesichert hatte.

Und um so schwerer traf uns der Schicksalsschlag, je plötzlicher, je unerwarteter er eintrat. Vor zwei Monaten erhielten wir die Kunde, dass Adolf $\mathrm{F}$ ischer wiederum von einer gefahrvollen Expedition in bis dahin unbekannte Gebiete des schwarzen Erdtheils glücklich zurückgekehrt, dass er - obwohl diese Reise nicht in 
jeder Hinsicht, nicht in gleicher Weise glicklich verlaufen, wie die früheren - wiederum grossartige wissenschaftliche Erfolge erzielt und reiche Sammlungen heimgebracht habe. Wenige Wochen später konnten wir ihn selbst begrüssen. Anscheinend gesund und frisch, wie wir ihn scheiden sahen, so stand er vor uns, voll Begeisterung seine neuen Entdeckungen darlegend, mit Lebhaftigkeit die überstandenen Gefahren schildernd, mit freudiger Hoffnung auf die baldige Bearbeitung seiner demnächst eintreffenden zoologischen Sammlungen hinweisend; - vierundzwanzig Stunden später pulsirte das Herz nicht mehr, welches so kraftvoll für die Erforschung Afrikas, für die Ornithologie geschlagen. Wir wähnten, dem verdienstvollen Reisenden, dem erfolgreichen Forscher die Ruhmeskrone zu reichen, - anstatt mit dem Eichenlaub sein Haupt zu schmücken, wanden wir für sein Grab den Immortellenkranz.

Gustav A dolf Fischer war am 4. März 1848 in Barmen geboren. Er besuchte zuerst das Gymnasium in seiner Vaterstadt, später in Köln, wo er das Abiturientenexamen mit Auszeichnung bestand. Schon während der Schulzeit trieb er mit Vorliebe Geographie und Naturwissenschaften und wusste in den Mussestunden an den wechselvollen Erlebnissen kühner Afrikaforscher für den dunklen Erdtheil sich zu begeistern. Nach Absolvirung der Schule (1869) wandte er sich dem Studium der Medizin und Naturwissenschaften zu, studirte in Bonn, Berlin und Würzburg, promovirte und legte an der letztgenannten Universität das Staatsexamen ab. 1874 trat er als Arzt bei dem 1. Garde-Dragoner-Regiment ein und wurde sodann dem in Emden garnisonirenden Ostfriesischen Infanterieregiment als Assistenzarzt überwiesen. 1876 erwirkte Dr. Fischer einen längeren Urlaub, um seinen von Jugend auf genährten Wunsch, eine Forschungsreise nach Afrika zu unternehmen, zur Ausführung zu bringen. Hiermit begann seine wissenschaftliche Laufbahn, auf welcher er durch rastlosen Eifer, durch Thatkraft, nie ermüdende Ausdauer und - was zur Erreichung hoher Ziele nicht fehlen darf - von einem Glücksstern begleitet, in kurzer Zeit die grossartigsten Erfolge errungen.

Um Vorbereitungen für die geplante Reise zu treffen, kam Dr. Fischer nach Berlin. In die Deutsche Ornithologische Gesellschaft eingeführt, gewann er bald ein lebhaftes Interesse für die Vogelkunde, und diese Disziplin blieb fortan sein Lieblingsfeld, welches er neben den allgemeinen geographischen Forschungen mit besonderer Aufmerksamkeit pflegte. Während mehrerer Monate 
studirte er täglich in der an afrikanischen Formen schon damals reichen Sammlung des Berliner Museums. Durch diese Studien in ornithologischer Hinsicht auf das gründlichste vorbereitet, verliess er Mitte November 1876 Europa und traf Anfang des Jahres 1877 in Sansibar ein.

Um eine Basis für fernere Operationen zu gewinnen, plante Fischer zunächst eine Expedition nach dem der Insel Sansibar gegenüber gelegenen Küstenstriche. Während der Vorbereitungen für diese Reise, der Anwerbung von Trägern und Führern, suchte er in der Suahelisprache sich zu vervollkommnen und benutzte die Mussestunden zu eingehender zoologischer Durchforschung der Insel Sansibar. Schon im April 1877 sandte er seinen ersten brieflichen Reisebericht, welcher im Journal für Ornithologie (1877 p. 171) abgedruckt ist. Es zeigte sich aus diesem Berichte sofort, wie gründlich Fischer für sein Unternehmen wissenschaftlich vorbereitet war, wie er schnell mit sachkundigem Verständniss unterschied, welche seiner Wahrnehmungen neu und wichtig für die Thierkunde seien, und wie er alles bereits Bekannte und Unwesentliche auszuscheiden wusste. Die nur flüchtig niedergeschriebenen Notizen seiner ersten Nachricht aus Afrika enthielten daher bereits eine Fülle neuer Thatsachen.

Im Juni 1877 trat $\mathrm{F}$ is cher seine erste Afrikareise an. Bis Ende des Jahres durchreiste er den Küstenstrich von Mombas bis Wito, vorzugsweise zoologisch sammelnd und beobachtend und mit ethnologischen Studien beschäftigt. Die ornithologischen Ergebnisse dieser Reise sind im Journal für Ornithologie 1878 p. 247 und f. und in den Reiseberichten ebenda 1877 p. 205 und 423 und 1878 p. 268, sowie im Ornithologischen Centralblatt 1878 p. 88 niedergelegt. 158 Arten wurden von $\mathrm{F}$ is cher während der Reise beobachtet und gesammelt, darunter vier neuentdeckte Formen, der prächtige Corythaix Fischeri, Euplectes diadematus, Coryphegnathus unicolor und Megalophonus Fischeri. Neben den werthvollen biologischen Beobachtungen, welche $\mathrm{F}$ is $\mathrm{ch}$ er's Sammlungen begleiteten, ist die sorgfältige Zubereitung der Objekte und peinlich gewissenhafte Etikettirung derselben hervorzuheben. Selten sind von einem Reisenden so saubere und in jeder Hinsicht musterguiltige Sammlungen heimgesandt worden.

Nach Sansibar zurückgekehrt rüstete sich Dr. F is ch er sofort zu einer grösseren Expedition in bisher noch unerforschte Gebiete. Bereits im Mai 1878 brach er in Begleitung der Gebrüder Den- 
hardt von neuem auf. Er durchzog zunächst die schon im Vorjahre bereisten Küstenstriche bis zur Mündung des Tana, verfolgte sodann diesen Fluss aufwärts behufs Feststellung des bis dahin unbekannten Laufes und erforschte das im oberen Gebiet des Tana gelegene Wapokomoland. Widerstand der Eingeborenen und schwere Erkrankung seiner Reisegefährten zwangen ihn nach mehrmonatlichem Aufenthalt in Wapokomo zur Rückkehr. Mit reichen wissenschaftlichen Schätzen kam er nach Sansibar. Er selbst hatte alle Strapazen ohne Nachtheil für seine Gesundheit überwunden, von dem Klima gar nicht gelitten. Die geographischen Erfolge der Wapokomoreise sind in den Mittheilungen der Geographischen Gesellschaft in Hamburg 1876/77 und 1878/79 eingehend besprochen. Ueberraschend war das ornithologische Ergebniss des Expedition. Dr. Fischer hatte Gebiete erschlossen, welche einen von den Küstendistrikten durchaus abweichenden faunistischen Character aufwiesen. Unter den 141 beobachteten Arten befanden sich nicht weniger als 20 bis dahin nicht bekannte species und darunter Formen, welche das grösste Aufseben erregten, wie Cosmopsarus regius, Speculipastor bicolor, Pachycoccyx validus u. a. Die ornithologische Ausbeute ist im Journ. f. Ornith. 1879 p. 337 u. f., 275 und 437 sowie im Ornith. Centralbl. 1879 p. 108, 114, 120, 139 und 155 beschrieben.

Um die Mittel für neue Reisen zu erwerben, fasste Fischer nunmehr den Entschluss, in Sansibar als Arzt sich niederzulassen. Sein bescheidenes, liebenswürdiges Wesen, seine Gewissenhaftigkeit, seine reichen Erfahrungen in der Behandlung der endemischen Krankheiten erwarben ihm schnell das Vertrauen, die Achtung und Zuneigung aller Kreise. Etwa vier Jahre, von 1879 bis 1882, war er in ärztlicher Praxis thätig. Während dieser Zeit setzte er seine zoologischen Studien und Sammlungen fort. Soweit sein ärztlicher Beruf dies gestattete, sammelte und beobachtete er theils selbst auf Sansibar oder auf kleineren Expeditionen, die er in die Küstenstriche des Festlandes unternahm, theils sandte er eingeborene Jäger mit besonderen Instructionen versehen in entferntere Gegenden. In diesen Zeitabschnitt fiel die Aufsehen erregende Entdeckung eines Flugeichhorns (Anomalurus orientalis) auf Sansibar und die Entdeckung mancher hochinteressanten neuen Vogelart, wie Linura Fischeri, Corythaix Reichenowi, Corythaix Cabanisi u. a. Das Journ. f. Ornith. 1880 p. 139 u. f., 187, 1883 p. 221, und das Ornith, Centralbl. 1880 p, 1 is u. 181 , 1882 p. 91 geben Nachricht 
über diese ornithologischen Forschungen. Gleichzeitig arbeitete Fischer den Plan zu einer dritten Reise aus, deren Ziel das bis dahin von keines Europäers Fuss betretene Massailand und der östlich des Victoria Njansa gelegene Mbaringo- und Samburu-See werden sollten.

Im Jahre 1882 hatte der unternehmende Forscher die Freude, auch dieses kühne Projekt der Ausführung nahe gerückt zu sehen. Ende dieses Jahres trat er im Auftrage der Geographischen Gesellschaft in Hamburg, welche die Kosten des Unternehmens trug, diese grosse und überaus wichtige Reise von dem Küstenort Pangani aus an. Er verfolgte das Thal des Pangani-Flusses aufwärts, dessen reiches Thierleben ihm Gelegenheit zu werthvollen zoologischen Beobachtungen lieferte, und wandte sich dann nordöstlich längs der Westseite des Pare-Gebirges und des Kilimandjaro in das Land der wilden Massai. Das Vordringen in diesem noch ganz unerforschten Lande war mit den grössten Schwierigkeiten verknüpft, la der Reisende kaum wagen durfte, von der Karawane oder aus den stets stark befestigten und bewachten Lagerstätten sich zu entfernen, ohne die Gefahr, von den umberstreifenden Massaikriegern niedergemetzelt zu werden. Mit Muth und Ausdauer alle Schwierigkeiten und Gefahren überwindend, gelang es dem kühnen Forscher, den Naiwascha-See, wenige Tagemärsche südlich des Mbaringo zu erreichen und auf einer stellenweise abweichenden Route nach sechs Monaten mit ausserordentlich wichtigen geographischen Resultaten und reichen ethnologischen und zoologischen Sammlungen glücklich zur Küste zurückzukehren. Gegen Ende des Jahres 1883 traf Dr. Fischer wieder in Europa ein. Nach siebenjähriger Abwesenheit konnten wir ihn in Berlin in der Decembersitzung 1883 der ornithologischen Gesellschaft zu seinen grossartigen Erfolgen beglückwünschen.

Die geographischen und ethnologischen Resultate der Massaireise sind von Dr. F is cher in den Mittheilungen der Geogr. Ges. in Hamburg 1882/83 bearbeitet worden. Eine englische Zeitschrift, das „Scot. Geographic. Magazine“ (1885) No. 9 sagte über diese Arbeit, insonderheit über die beigegebene, von dem Reisenden aufgenommene Karte der durchforschten Gebiete: ,Wir beglückwünschen Dr. Fischer zu der erfolgreichen Art, wie er die Landstrecken kartographisch behandelt hat, welche er durchwanderte. Es ist lange her, dass wir eine Arbeit gesehen haben, in welcher so sorgsam und genau und mit so echt wissenschaftlicher Methode 
geographische Beobachtungen aufgezeichnet wären." Wenn man erwägt, wie zurückhaltend die Engländer mit Anerkennung Ausländern gegenüber sich stets verhalten, so darf man mit Recht Werth auf dieses Urtheil legen, welches dem Werke unseres verstorbenen Freundes so auszeichnendes Lob spendet.

Die zoologischen Sammlungen der Massaireise brachten uns wieder eine ungeahnte Fülle neuer Formen. Neben einer grossen Reihe neuer Säugethiere, Reptilien, Insekten enthielten die Sammlungen 36 neue Vogelarten, darunter Lusciola africana, Parus fringillinus, Euplectes Friederichseni, Nigrita Cabanisi, Notauges Fischeri, Prionops poliolophus, Drepanorhynchus Reichenowi als die auffallendsten. Die in Madarasz Zeitschr. f. Ornith. veröffentlichte Uebersicht weist 345 während der Reise beobachtete Vogelarten auf. die neuen Species wurden im Journal f. Ornith. 1884 p. 52, 178 und 260 beschrieben.

Ein und ein halbes Jahr lang hatten wir die Freude, Dr. Fis ch er unter uns zu sehen. Manche Sitzung unserer Gesellschaft ist mit Vorlagen, mit Besprechung seiner ornithologischen Entdeckungen ausgefüllt worden. Eine zusammenfassende Uebersicht der ornithologischen Resultate seines siebenjährigen Aufenthaltes in Afrika hat F is cher im Journal f. Ornith. 1885 p. 113 u. f. veröffentlicht. Dieselbe weist 479 von ihm an zahlreichen Punkten eines über 7-8 Breiten- und ebensovielen Längengrade sich erstreckenden Gebietes gesammelte Vogelarten und unter diesen 70 neu entdeckte Species auf. Noch niemals hat ein Afrikareisender einen gleichen ornithologischen Erfolg errungen, und man kann mit grösster Wahrscheinlichkeit behaupten, dass ein gleiches Ergebniss in Zukunft nie wieder erreicht werden wird.

Nicht lange duldete es den rastlosen Forscher in Europa. Nachdem er die Folgen der anstrengenden Massaireise, auf welcher er mehr als bei den früheren Expeditionen von den Einflüssen des feindlichen Klimas zu leiden gehabt, von einem Gallenfieber ergriffen sogar in grösster Lebensgefahr geschwebt, überwunden hatte, sehnte er sich nach neuen Unternehmungen. Mit Freude ergriff er das Anerbieten, welches von der Familie des damals seit mehreren Jahren verschollenen Afrikareisenden Dr. Junker ihm gestellt wurde, zur Aufsuchung, nöthigenfalls zur Befreiung dieses Forschers eine Expedition nach dem Victoria Njansa zu unternehmen und von dort das Vordringen in die oberen Nilgegenden zu versuchen. 
Von unserm "Glück auf" begleitet verliess Dr. Fischer im Mai 1885 zum zweiten Male Europa. Es waren ihm 40000 Mark zur Verfügung gestellt. Obwohl er erkannte, dass diese Mittel zur Ausrüstung einer Karawane von mehreren Hundert Mann, wie sie die geplante Reise erforderte, und zur Durchführung des schwierigen Unternehmens nicht ausreichen würden, zögerte er doch nicht, die Expedition anzutreten, da jeder Aufschub dem gesuchten Reisenden Gefahr bringen konnte, und ergänzte aus eigenen Mitteln das Fehlende. Am 1. August 1885 brach er von Pangani auf. Auf grösstentheils von Europäern noch nicht betretenen Wegen zog er durch die Landschaften Ungu, Kibaia, Irangi und Ussandavi und erreichte glücklich den Ort Kagehi an der Südseite des Victoria-Sees. In der Absicht, über den See zu fahren und nordwärts weiter vorzudringen, schickte er Boten an den Nachfolger des leider verstorbenen, den Europäern freundlich gesinnten Königs Mtesa von Uganda, um die Erlaubniss auszuwirken, sein Land zu durchziehen. Der neue, den Weissen feindliche Sultan verweigerte die Erlaubniss. Ein Brief des englischen Missionars Makay in Uganda warnte $\mathrm{F}$ is cher dringend vor einem Versuch trotz des Verbotes Uganda zu betreten, da der Expedition dasselbe Ende bereitet werden würde, wie dem kurz vorher mit 32 Leuten ermordeten englischen Bischofs Hannington. Hiermit war der Erfolg seiner Expedition entschieden. Derselbe wurde ausschliesslich auf die Möglichkeit eines Durchzuges durch Uganda begründet. Das Scheitern dieser Hoffnung bedeutete die Rückkehr. F is ch er's Muth und Thatkraft sträubten sich gegen diese Nothwendigkeit. Er wollte das vorgezeichnete Ziel nicht aufgeben, bevor alle Möglichkeiten des Gelingens erschöpft waren. Obwohl die mitgeführten Waaren ausschliesslich mit Rücksicht auf die geplante Route ausgewählt waren und für andere Gebiete nicht geeignet erschienen, obwohl die vorhandenen Vorräthe überhaupt für eine längere Reise nicht ausreichten, wagte $\mathrm{F}$ ischer dennoch den Versuch, den Victoria See im Osten zu umgehen, um durch das I and der Kawirondo in das Nilgebiet und in deu Bereich des Gesuchten vorzudringen. In Kawanga nordöstlich des Victoria Njansa hoffte er im Austausch gegen Munition Lebensmittel und damit die Möglichkeit des weiteren Vordringens erlangen zu können. Auch diese Hoffnung schlug fehl. Hungersnoth war ausgebrochen, und die Expedition musste, in kärglichster Weise ihr Leben fristend, weiter östlich über den Mbaringo- und Naiwascha See, Kikuja 
und Ukamba zur Küste zurückkehren, welche bei Wanga erreicht wurde. Unsägliche Strapazen und Entbehrungen legte diese Reise der Expedition auf, die höchsten Anforderungen stellte sie an deren Führer. Aber mit der ihm eigenen Zähigkeit und Energie wusste Dr. Fischer alle Schwierigkeiten zu überwinden. Von seinen 220 Mann hatte er 40 durch Hunger, Krankheiten und Verletzungen bei mehreren Zusammenstössen mit feinlichen Eingeborenen verloren. Er selbst hatte allen Anstrengungen getrotzt. Anscheinend nicht berührt durch die Strapazen kehrte er zurück.

War es ihm diesmal durch die Ungunst der Verhältnisse auch nicht gelungen, das eigentliche Ziel, die Aufsuchung des verschollenen Dr. Junker, zu erreichen, so hatte die Reise doch wiederum wichtige geographische und zoologische Resultate geliefert, wie die Entdeckung neuer Quellflüsse des Nil im Osten des Victoria-Njansa, die Erweiterung der östlichen Grenze des westafrikanischen Faunengebietes bis zum Victoria und anderes, worüber erst die Bearbeitung seiner Tagebücher und der glïcklich mitgeführten Sammlungen vollständigen Aufschluss geben wird.

Ende September 1886 traf Dr. Fischer in Europa ein. Während des Octobers hielt er sich im Hause seiner Eltern in Düsseldorf auf und beschäftigte sich mit der Abfassung eines vorläufigen Berichtes über seine Reise, welcher für die „Geographischen Mittheilungen" bestimmt war. Auf Einladung der Geogr. Gesellsch. in Hamburg hielt er in der Novembersitzung derselben einen Vortrag über die geographischen Resultate der letzten Expedition. Von Hamburg kam er am 8. November nach Berlin, in der Absicht, das Eintreffen seiner zoologischen Sammlungen hier zu erwarten, um dann sofort an die Bearbeitung derselben zu gehen. Nichts deutete äusserlich auf eine Erkrankung innerer Organe. Er selbst, der durch seine Reisen und seinen langen Aufenthalt in feindlichem Klima gewohnt war, mit Sorgfalt sein körperliches Befinden zu überwachen, der als Arzt, insonderheit durch seine afrikanische Praxis mit den Symptomen der tropischen Krankheiten genau vertraut war, fühlte keinerlei Unbehagen, äusserte nicht die geringste Besorgniss, dass die Anstrengungen der letzten Reise nachtheilige Folgen für seine Gesundheit hinterlassen haben könnten. Trotzdem trug er den Todeskeim bereits in sich. In der Nacht vom 9. zum 10. November befiel ihn plötzlich ein Gallenfieber; - am Mittag des 11. November, an demselben Tage, an welchem vor zwei Jahren der unvergessliche $\mathrm{Br}$ eh m uns entrissen wurde, war er entschlafen 
In kurzen Zügen und in schlichter Form - denn zur Schilderung wahren Verdienstes bedarf es keines Wortgeklingels - ist vorstehend der wissenschaftlichen Wirksamkeit Dr. F is cher's gedacht. Nicht unerwähnt darf bleiben, dass er der erste war, welcher auf die Bedeutung Ost-Afrikas als Kolonialbesitz für Deutschland hingewiesen. In seinen Privatbriefen von 1879 und später wiederholt sich die Mahnung, zuzugreifen, da es noch Zeit sei, und für die Zukunft vielversprechende Kolonien dem deutschen Reiche zu sichern. Somit ein warmer Freund der deutschen kolonisatorischen Bestrebungen in Ostafrika, sah er sich dennoch später veranlasst, gegen diese Unternehmungen öffentlich aufzutreten, als begründete Befürchtung vorlag, dass dieselben in unrichtige Bahnen geleitet würden. Sicherlich hat seine Arbeit „Mehr Licht im dunklen Welttheil", welche auf Grund der langjährigen Erfahrungen die eingehendste Schilderung der Ostafrikanischen Verhältnisse liefert, die wir überhaupt besitzen, dazu beigetragen, dass das grossartig und kühn angelegte Werk jetzt wieder in das richtige Fahrwasser gelangte.

Ist es uns gestattet, noch persönlicher Eigenschaften des Dahingeschiedenen zu gedenken, so dürfen wir seine Selbstlosigkeit, seine Anspruchslosigkeit und Bescheidenheit rühmen. Niemals trat er mit seiner Person in den Vordergrund. Sein Leben gehörte voll und ganz der Wissenschaft, für seine Person erstrebte er nichts. Wohl glitt ein wohlgefälliges Lächeln über sein Gesicht, wenn wir mit ihm die werthvollen Sammlungen auspackten, die ausgebreiteten Schätze ordneten, und die Ueberraschung in lautem Beifall sich äusserte; aber in diesem Lächeln spiegelte sich nur die harmlose innere, man darf sagen kindliche Freude an den herrlichen Errungenschaften. Niemals offenbarte sich die geringste Eitelkeit, niemals ein wohlberechtigter Stolz über die eigenen Leistungen. Liebenswürdig, gefällig, stets bereit, auf abweichende Ansichten oder Vorschläge einzugehen, dabei doch voller Charakterfestigkeit und nur nach eigener sorgfältiger Prüfung alles Vorgenommene regelnd, so steht Adolf $\mathrm{Fischer}$ in unserer $\mathrm{Er}$ innerung.

Die Wissenschaft hat - zu früh - einen ihrer eifrigsten und erfolgreichsten Förderer verloren. Die Deutsche Ornithologische Gesellschaft beklagt den Tod eines ihrer thätigsten Mitglieder. Wir Alle, die wir dem Dahingeschiedenen nahe gestanden, werden 
dem treuen Freund und Genossen eine dankbare Erinnerung, seinem hochherzigen Streben ehrenvolle Anerkennung bewahren. Friede seiner Asche!

Anton Reichenow.

\section{Notizen. Ueber Cuculus canorus ete.}

I.

In einem Weinbergsgrundstïcke nahe der hiesigen Stadt hatte im vorigen Jahre ein Hänflingspärchen sich einen Stachelbeerstrauch als Nistplatz gewählt und bereits vier Eier in das Nest eingelegt, als seltsamerweise ein Kukuksweibchen sich diese körnerfressenden Brutvögel als Pflegeeltern ausersehen und ihr Ei zum Mitausbriten in jenes Nest eingeschmuggelt hatte. Diese Pflegeeltern scheinen jedoch nicht gewillt gewesen zu sein, einen Fremdling zu erziehen, denn sie verliessen das Nest mit dem Gelege, um sich vielleicht anderwärts sesshaft zu machen. Als hierauf der Bewohner des Grundstïcks, welcher mit der sorgfältigen Beaufsichtigung und Ueberwachung dieser Niststätte beauftragt worden war, den Inhalt des Nestes besichtigte, fand er, dass die verlassenen 4 Eier des Hänflings noch unversebrt vorhanden waren, während das Kukuksei aus dem Neste verschwunden war. Kurz vorher will er den Kukuk in der Nähe des Nistplatzes bemerkt haben. Es fragt sich nun: Hat das Kukuksweibchen ihr Ei aus dem verlassenen Nestgelege fortgenommen, um es vielleicht einem andern Brutvogel unterzuschieben, oder hat sie es vernichtet? Dass das Kukuksei durch irgend ein Raubthier aus dem Neste geholt worden sein könne, lässt sich nicht füglich annehmen, weil dasselbe sich mit dem Kukuksei allein - und warum gerade mit diesem - nicht begnügt, sondern unzweifelhaft auch die vier Nesteier mit geraubt haben würde. Eben so wenig kann das Kukuksei durch Menschenhände entfernt worden sein, weil während der kurzen Lege- und Brlitezeit des Hänflings fremde Personen jenes Grundstück nicht betreten haben. Also muthmasslich eine, meines Wissens neue, das ornithologische Interesse erregende Eigenthümlichkeit des Kukuks in seinem Leben und Treiben.

II.

Nach den angestellten Beobachtungen haben sich seit einigen Jahren in hiesiger Gegend verschiedene Zugvögel auffallend ver- 


\section{$2 \mathrm{BHL}$ Biodiversity Heritage Library}

1886. "Zur Erinnerung an Gustav Adolf Fischer." Journal fu

r Ornithologie 34, 613-622. https://doi.org/10.1007/bf02008700.

View This Item Online: https://www.biodiversitylibrary.org/item/101744

DOI: https://doi.org/10.1007/bf02008700

Permalink: https://www.biodiversitylibrary.org/partpdf/142894

\section{Holding Institution}

Smithsonian Libraries

\section{Sponsored by}

Biodiversity Heritage Library

\section{Copyright \& Reuse}

Copyright Status: Public domain. The BHL considers that this work is no longer under copyright protection.

This document was created from content at the Biodiversity Heritage Library, the world's largest open access digital library for biodiversity literature and archives. Visit BHL at https://www.biodiversitylibrary.org. 